\title{
Bone and Joint Tuberculosis in the UK: delays in diagnosis and treatment
}

\section{Abstract}

Aims: Tuberculosis (TB) infection of bones and joints accounts for $6.7 \%$ of TB cases in England and is associated with significant morbidity and disability. Public Health England reports that TB patients experience delays in diagnosis and treatment. This study's objectives were to determine the demographics, presentation and investigation of bone and joint TB infections, to assist doctors assessing potential cases and identify avoidable delays.

Patients and Methods: Retrospective observational study of all adults with positive TB cultures on specimens taken at a tertiary orthopaedic centre over two years. A laboratory information system search identified subjects. Demographics, clinical presentation, radiology, histopathology and key clinical dates were obtained from medical records.

Results: Thirty-one adults were TB culture-positive: median age 37 years; $68 \%$ male; $90 \%$ were migrants. Main sites affected were joints (32\%), the spine (26\%) and long bones (19\%); $26 \%$ had multifocal disease. The most common presenting symptoms were pain (94\%) and swelling (93\%); "typical" TB symptoms, such as fever, sweats and weight loss were uncommon. Patients waited 7 months between symptom-onset and referral to the tertiary centre and 68 days from referral to starting treatment. Radiology suggested TB in $84 \%$, but in $19 \%$ of these initial biopsy specimens were not sent for mycobacterial culture, necessitating a second biopsy. Rapid PCR-based testing for TB using Xpert MTB/RIF ${ }^{\circledR}$ was performed in 5 subjects; $80 \%$ tested positive for TB. These patients had reduced time from their diagnostic biopsy to starting treatment than those whose samples were not tested ( 8 days versus 36 days, $p=0.016$ ).

Conclusion: Patients with bone and joint TB experience delays in diagnosis and treatment, some of which are avoidable. Maintaining a high index-of-clinical-suspicion and sending specimens for mycobacterial culture are crucial to avoid missing cases. Rapid diagnostic tests reduce delays-to-treatment and should be performed on patients with radiological features of TB. 


\section{Introduction}

Since 2011, there has been a year-on-year decline in the incidence of tuberculosis (TB) in England, with the number of cases falling from a peak of 15.6 per $100,000(8,280$ cases) in 2011 , to 10.5 per $100,000(5,758$ cases $)$ in 2015 . The proportion of extrapulmonary TB cases has remained constant (46.6\% in 2015), and is higher in those born outside of the UK (53.1\% of total TB cases) compared to those who are UK-born (30.1\%). Bone and joint TB (BJTB), which includes spinal TB, accounts for $6.7 \%$ of England's total TB cases and $14.4 \%$ of its extra-pulmonary cases ${ }^{1}$. BJTB is associated with significant morbidity including chronic pain, deformity and disability ${ }^{2}$ so early diagnosis and treatment is desirable.

Delays of weeks before starting TB treatment is common for patients: in England, 28\% of patients with pulmonary TB experience delays of four months or longer between symptom-onset and starting treatment ${ }^{1}$. European studies of spinal TB also report diagnostic delays ${ }^{3-5}$. With just 386 cases of BJTB notified in $2015^{1}$, it is a condition rarely seen by surgical teams, particularly in non-specialist centres outside London. Inexperience with its clinical presentation and diagnostic work-up and its varied presentation at different anatomical sites risks these patients experiencing long delays before starting treatment, which risks clinical deterioration and worsening disability 6 .

We performed this retrospective observational study of BJTB aiming to describe the typical demographics, presentation and investigation of these patients, to assist doctors assessing potential cases. We investigated the clinical pathways through which diagnosis was confirmed and treatment started, aiming to identify avoidable delays whose prevention might reduce time-to-treatment.

\section{Patients and Methods}

We conducted this study at a large specialist orthopaedic hospital in the UK which includes a regional Sarcoma Unit, accepting regional referrals for patients with possible sarcoma. Bone and joint samples were cultured for TB using the BACTEC MIGIT system (BD, Franklin Lakes, New Jersey, USA). Suspected mycobacterial isolates were sent to Public Health England's (PHE) Mycobacterial reference lab for confirmation, speciation and sensitivity-testing. Rapid Polymerase Chain Reaction (PCR)-based testing was performed locally using the Xpert MTB/RIF ${ }^{\circledR}$ (Cepheid, Sunnyvale, California, USA) assay.

We included all patients aged 18 years and over who had pathological specimens taken at the centre between $1^{\text {st }}$ June 2012 and 30 ${ }^{\text {th }}$ May 2014 which grew Mycobacterium tuberculosis (Mtb). Subjects were identified via a laboratory information search system (WinPath). Paper and electronic records were accessed with appropriate permissions. A standardised proforma recorded: demographics, clinical presentation, radiological findings, histopathology and important dates (including first presentation, referral to the specialist orthopaedic centre, biopsy, results, communication of results to the GP and/or 
referring hospital, referral to TB services, commencement of anti-tuberculous antimicrobials). Data were anonymised and recorded in Excel (2010).

Analysis

Data were analysed using Microsoft Excel 2010 and GraphPad 2017 (GraphPad Software, Inc. California, USA). After descriptive study of the different variables, MannWhitney $U$ test was applied for comparison of medians. Tests were two-tailed; a P-value of 0.05 was considered significant.

\section{Results}

During the study period, cultures from 31 adult patients were positive for TB. All were drug-susceptible, meaning sensitive to first-line antibiotics (Rifampicin, Isoniazid, Pyrazinamide and Ethambutol).

\section{Demographic characteristics}

Subjects' median age was 37 years (IQR 29-53 years); 68\% were male $(n=31)$. Ninety percent $(n=28)$ were born outside the UK, predominantly in southern Asia (Table 1), living in the UK for a median of 6 years at the time of diagnosis (IQR 3.5-14 years, $n=25$ ). Three subjects were UK-born; their median age was 41, range 29 to 85 years; their selfreported ethnicities were "White British", "Pakistani "and "Black-other". No patients were known to have Human Immunodeficiency Virus (HIV) prior to diagnosis. One patient was tested for HIV at the orthopaedic centre and tested negative.

\section{Site of infection and clinical features}

The most commonly affected sites were joints $(32 \%, n=31)$ and the spine $(26 \%)$, followed by long bones (19\%) and axial bones (13\%). Multifocal disease, defined as more than one anatomical site (bones, joints or elsewhere), was present in 26\%; 6 of these 8 cases had spinal involvement (Table 2). Commonly reported symptoms were pain $(94 \%, 29 / 31)$ and swelling $(93 \%, 26 / 28)$ but "typical" TB symptoms were uncommon, with $21 \%$ (3/14) reporting fever, 14\% (2/14) night sweats and 56\% (9/16) weight loss. Few were asked about TB contacts (7/31) or past infection (1/22 patients). Where this information was available, three reported a TB contact and one reported a prior history of TB.

\section{Treatment delays and Referral pathways}

Median time from symptom-onset to starting anti-tuberculous therapy was 9 months (IQR 6-20 months, $n=26$ ) with $37 \%$ waiting one year or longer. We examined the referral, diagnostic and treatment processes in further detail, to identify potentially avoidable areas of delay in the patients' pathway between symptom-onset and starting treatment.

Median time from symptom-onset to referral to the tertiary centre was 7 months (IQR 3-13.5 months, $n=21$ ). There was insufficient data to establish what proportion of this 
time was attributable to the patient not seeking medical advice, and what proportion was spent being managed in primary or local secondary care. Ninety percent $(28 / 31)$ were referred to the tertiary centre via the sarcoma pathway and all of these were seen within the two-week target for suspected cancer referrals. Referral data were available for two of the three non-sarcoma referrals; they were seen in clinic 27 and 33 days after referral. Median time between referral to the specialist centre and starting TB treatment was 68 days (IQR 47-103 days, $n=30$ ); 33\% waited 3 months or longer; 10\% waited 6 months or longer.

After clinical review, $97 \%$ of patients underwent Magnetic resonance imaging (MRI). It was contraindicated in one patient who had a CT scan. In $84 \%$ of cases, the radiologist reported TB in their differential diagnosis based on the imaging, prior to biopsy $(n=31)$. All patients underwent a biopsy: $92 \%$ had specimens sent for histopathology and $77 \%$ for TB culture $(n=31)$. Seven patients' samples from their first biopsy $(23 \%)$ were not sent for TB culture; TB was suspected in five of these; all seven required a second biopsy. Median time to the second biopsy was 39 days (IQR 19-52 days, $n=7$ ).

\section{Pathology results}

Granulomas were seen in $87 \%$ (20/23) of histopathology samples; no acid-fast bacilli (AFB) were seen $(n=25)$. TB cultures were positive in $92 \%(22 / 24)$ of first biopsies; median time to culture positivity was 22 days (IQR 16-38 days, $n=31$ ); plus 10 days (IQR 9-16 days, $n=31$ ) for confirmation of $M t b$ species Xpert MTB/RIF ${ }^{\circledR}$ was performed on five patients' samples, taking two days from biopsy to results (IQR 2-7 days, $n=5$ ); four tested positive for $M t b$ and negative for rifampicin-resistance. One patient started antituberculous treatment before their biopsy; for the remaining 30 patients, those whose samples were tested with Xpert MTB/RIF ${ }^{\circledR}$ waited significantly less time from their diagnostic biopsy to starting treatment than those whose samples were not tested (median 8 days with Xpert MTB/RIF ${ }^{\circledR}, n=5$, versus 36 days without, $n=25, p=0.016$ ).

\section{Starting treatment}

Anti-tuberculous treatment was started in 13 patients prior to TB cultures becoming positive. For the remaining 18, median time from culture-positivity to treatment was 20 days (IQR 9-33 days, $n=18$ ). Of these 18, 10 were referred onwards prior to culturepositivity (to TB clinic (5), the GP (4), referring hospital (1)). Referral-to-treatment time was available for $4 / 6$ who were referred after cultures grew TB: median time was four days for the three referred directly to TB clinic; one patient was referred to the GP and waited 49 days before starting treatment in TB clinic.

\section{Discussion}

Diagnosing BJTB is challenging and there is no specific national guidance on the optimal diagnostic pathway. In this study of culture-positive BJTB, patients waited an average of 9 months between symptom-onset and starting anti-tuberculous therapy, substantially longer than the national average time for pulmonary cases of 74 days ${ }^{1}$ and longer than 
reported in previous European studies on spinal TB. ${ }^{3-5}$. Seven of these months occurred prior to referral to the specialist centre. We could not determine what proportion of this time was attributable to delays in patients engaging with medical care, and what was attributable to patients being managed within primary and secondary care, but the nonspecific clinical presentation of BJTB is likely to have been a contributing factor. Omitting to send biopsy specimens for TB culture contributed a further 5 weeks to delays; waiting for TB culture results took 3 to 4 weeks; not referring directly to TB clinic may have also contributed to delays. Maintaining a high index-of-clinical-suspicion, targeted use of rapid molecular tests and direct patient referral to TB services should reduce time-totreatment in these patients.

\section{Clinical presentation}

In this BJTB cohort, patients were typically young male migrants from southern Asia. This is in keeping with national data from PHE: 73\% of patients diagnosed with TB in 2015 were born outside of the UK, most commonly India, Pakistan and Bangladesh ${ }^{1}$. Previous studies on BJTB in low-endemic countries report varying proportions of cases occurring in immigrants, with some centres reporting high proportions (92\% UK ${ }^{3} ; 83 \%$ Denmark $^{7}, 66 \%$ Netherlands $^{8}$ ) and others in France ${ }^{9}$ and Spain $^{5}$ reporting much lower figures. Countries-of-origin of affected immigrants vary in these studies, likely reflecting differing migration trends.

Few patients presented with "typical" TB symptoms of fevers and night sweats and just over half reported weight loss. INon-specific symptoms of pain and swelling were very common,consistent with previous studies ${ }^{3,5,7}$, meaning clinicians cannot rely on clinical presentation alone to suggest underlying TB. Maintaining a high index-of-suspicion and knowledge of local epidemiology and high-risk groups are essential to ensure the necessary microbiological tests are requested and the diagnosis is not missed.

The spine is usually the most common site of BJTB (65\% in PHE national data ${ }^{1}, 54.3 \%$ in a Danish cohort ${ }^{7}$ ) but just $26 \%$ of our cohort had spinal involvement. This distribution likely reflects the fact that $90 \%$ of our patients were referred via the sarcoma pathway and the centre does not take emergency admissions. Clinically, TB can mimic sarcoma: both diseases can be insidious, affect younger adults and present with swelling and pain ${ }^{10}$. European studies have reported up to $35 \%$ of BJTB patients as having concomitant pulmonary $\mathrm{TB}^{3,7,9}$. While $26 \%$ of our cohort had multifocal disease, none had pulmonary involvement.Acutely unwell patients with pulmonary involvement would likely have presented via alternative pathways so would not have been included in this study. .

\section{Diagnosis}

In the majority of cases, radiological and histopathological findings suggested underlying TB. MRI was very useful, with TB considered in $83 \%$ of MRI reports. Data showing what proportion of radiology reports overall list TB in the differential diagnosis were 
unavailable. Radiological features of BJTB are diverse and non-specific. In the spine, TB usually affects multiple anterior vertebral bodiesand adjacent discs; paraspinal abscesses are common. Vertebral collapse and anterior wedging are seen in advanced infection. TB infection of the bone causes a chronic osteomyelitis, so radiological features are non-specific. Similarly tuberculous arthritis resembles other infectious and inflammatory arthritides ${ }^{2,11}$.

Typical histopathological features of TB include granulomas and AFB ${ }^{12-13}$. Granulomas were seen frequently in this study: their presence provides strong evidence for underlying TB, useful when TB cultures are negative. Conversely, AFB were not seen in any of our cases. Visible only when mycobacterial burden is high and affected by formalin ${ }^{14}$, their absence should not deter the clinician from diagnosing TB.

Every patient in this study underwent a biopsy but $23 \%$ did not have samples sent for mycobacterial culture, despite TB being in the differential diagnosis for most of them. All seven had to undergo a second biopsy and waited an average of five weeks for the procedure. Biopsy is crucial for confirming a diagnosis of TB. All efforts should be made to obtain tissue specimens and send them to histopathology and microbiology. Microbiological specimens must be sent formalin-free ${ }^{15}$, as formalin sterilises any organisms present, rendering cultures falsely negative. National Standards for Microbiology recommend that routine mycobacterial culture is considered for all nonpostoperative spinal infections ${ }^{16}$. We recommend that mycobacterial culture is requested for any patient in whom infection or malignancy is suspected, or where there is clinical uncertainty. This recommendation has been implemented in local policy since the study period.

Mycobacterial culture is essential for obtaining drug-susceptibility information and is another important reason for performing a biopsy whenever possible. While no patients in this study had drug-resistant strains, $7.4 \%$ of England's TB cases are resistant to at least one first-line antibiotic and $1.3 \%$ are "Multi-drug resistant" (MDR), meaning the isolate is resistant to both isoniazid and rifampicin ${ }^{1}$. Worldwide, $5 \%$ of TB cases are MDR, with rates over $20 \%$ in some ex-Soviet states ${ }^{17}$. In England, most MDR-TB cases are imported from Latvia (20\%) and Lithuania $(23.9 \%)^{1}$.

\section{Rapid Molecular Tests}

While mycobacterial culture remains the gold standard for diagnosing tuberculosis ${ }^{18}$, its primary disadvantage is the time it takes to grow TB: median time to culture positivity in this study was 22 days. Five patients' samples were tested with Xpert ${ }^{\circledast}$ MTB/RIF; 80\% returned a positive result, with an average turn-around time of two days and those whose samples were tested with Xpert ${ }^{\circledR}$ MTB/RIF waited four weeks less from their diagnostic biopsy to starting treatment than those whose samples were not tested.

Xpert ${ }^{\circledR}$ MTB/RIF is a PCR-based assay that allows the rapid diagnosis of TB within just a few hours. It was initially developed for use with sputum to diagnose pulmonary TB, 
where its sensitivity approaches $100 \%$ for smear-positive disease ${ }^{19}$. The World Health Organisation (WHO) recommends $\mathrm{Xpert}^{\circledR} \mathrm{MTB} / \mathrm{RIF}$ for testing non-respiratory samples in patients suspected of having extra-pulmonary $\mathrm{TB}^{20}$. Its sensitivity in tissue ranges from $77 \%$ to $90 \%$ (specificity $96-98 \%$ ) when compared to mycobacterial culture, with the highest sensitivities in lymph node samples. In pus, sensitivities range from $77 \%$ to $96 \%$ (specificity $46 \%-86 \%$ ); lower sensitivities are reported with fluid samples (sensitivity $50-76 \%)^{21-23}$. In BJTB studies, sensitivities of $96 \%$ (spinal TB, South Africa) ${ }^{24}$ and $100 \%$ (BJTB, China) ${ }^{25}$ have been reported. Xpert ${ }^{\circledR}$ MTB/RIF also rapidly detects rifampicinresistance with a sensitivity approaching $100 \%{ }^{19}$, making it an invaluable tool when investigating patients from high-risk countries for MDR-TB.

GenoType MTBDRplus ${ }^{\circledR}$ (Hain Lifescience $\mathrm{GmbH}$, Nehren, Germany) is another molecular test which can provide rapid confirmation of the presence of Mtb DNA, and common mutations associated with rifampicin- and isoniazid-resistance. Results are typically available within two to three days and reported sensitivities in pulmonary samples are comparable to Xpert ${ }^{\circledR}$ MTB/RIF $^{26}$. GenoType MTBDRplus ${ }^{\circledR}$ was assessed in the Chinese BJTB study and found to have a sensitivity of $87.5 \%$ for culture-confirmed cases and $63.6 \%$ for culture-negative "probable" cases ${ }^{25}$.

Almost all hospitals have access to Xpert ${ }^{\circledR}$ MTB/RIF and GenoType MTBDRplus ${ }^{\circledR}$, though most microbiology departments do not offer it "in-house" 27 . The positive predictive value of the test is affected by the prevalence of TB. Therefore, in low prevalence countries such as the UK, they should not be used indiscriminately due to the risk of false-positive results. Where clinical suspicion and therefore pre-test probability is high, their use can reduce time-to-diagnosis and treatment from weeks to days. Requesting doctors should be aware of the possibility of false-negatives and should not be deterred by a negative PCR in a high-risk patient. We recommend considering rapid molecular testing for samples from all patients where BJTB and sarcoma are in the differential diagnosis and are changing local policy to reflect this. Given the importance of targeted testing with these assays and their interpretation, we recommend involving an Infection specialist early.

Initiating anti-tuberculous treatment and referral to TB services

Over $40 \%$ of patients in this study started TB treatment before positive culture results were available. Where the patient is very symptomatic and the index-of-suspicion is high, this is a reasonable approach that should be made in partnership with a TB specialist. However, unless there is a clinical indication to start treatment urgently, antibiotics should not be initiated until after a biopsy has been performed and samples sent for mycobacterial culture. This will maximise the likelihood of growing TB from the tissue sample, which will enable confirmation of the diagnosis and drug-susceptibility testing.

The patient's local TB clinic is responsible for overseeing their anti-tuberculous treatment, which in most cases will be a 6 -month course ${ }^{18}$, and initiating contact 
tracing. In this study, there was variation in how and when patients were referred to TB services. Numbers are too small to make a meaningful comparison, but where the TB diagnosis is confirmed, we recommend referring patients directly to their local TB service to avoid unnecessary delays.

\section{Limitations}

This study was conducted in a tertiary centre which receives UK-wide referrals, so it manages more BJTB cases than most. Its role as a sarcoma centre likely distorts its casemix as seen with the under-representation of spinal TB, but many of our findings and recommendations will be applicable to general orthopaedic units.

In this study, most patients had been symptomatic for months before referral to the tertiary centre. Unfortunately there was insufficient data to establish what proportion of this time was attributable to the patient not seeking medical advice and what was attributable to time being managed in primary or local secondary care. If the latter were a significant contributing factor, additional training of general practitioners and hospital doctors might be beneficial. There was insufficient information in medical records to establish why some patients with suspected BJTB were not started on treatment until after culture results were available.

Mycobacterial culture is not $100 \%$ sensitive ${ }^{13}$ : nationally $45.9 \%$ of extra-pulmonary TB cases are culture-confirmed (compared to $72.7 \%$ of pulmonary cases) ${ }^{1}$. Our study includes only patients with culture-positive BJTB and therefore misses those treated presumptively for BJTB without positive cultures. Many patients at our centre are referred to and from other hospitals and their ultimate diagnosis is not always available locally. As we could not reliably capture this group, they were not included in this study.

As with any retrospective study, some data are incomplete despite our best efforts. We are confident that our Winpath system search captured all cases of culture-positive BJTB diagnosed during the study period.

\section{Conclusions}

Patients with BJTB typically wait months for diagnosis and treatment and some of these delays are avoidable. BJTB's non-specific presentation means that an index-of-suspicion must be maintained to avoid missing cases, particularly in high-risk patients (young, male, recent migrants) with bone and joint lesions. Mycobacterial culture of biopsy specimens is crucial for diagnosis and drug-susceptibility testing and should be performed in any case where infection or malignancy is being considered or when there is clinical uncertainty. Early involvement of an Infection Specialist is recommended: they can advise on and expedite microbiological testing and access specialist diagnostic tests such as $\mathrm{Xpert}^{\circledR}$ MTB/RIF. The use of rapid diagnostic tests such as Xpert ${ }^{\circledR}$ significantly $^{\circledR}$ speeds up diagnosis and starting anti-tuberculous therapy. They can also advise on antibiotic treatment and facilitate engagement with local TB services. On diagnosis, the managing team should refer the patient directly to their local TB service. 


\section{$\underline{\text { References }}$}

1. Public Health England. Tuberculosis in England 2016 report. London, 2016.

2. Pigrau-Serrallach and Rodríguez-Pardo. Bone and joint tuberculosis. Eur Spine J 2013;22(Supp4):S556-S566.

3. Kenyon PC, Chapman AL. Tuberculous vertebral osteomyelitis: findings of a 10-year review of experience in a UK centre. J Infect 2009;59:372-3.

4. Luzzati R, Giacomazzi D, Danzi MC, et al. Diagnosis, management and outcome of clinically suspected spinal infection. J Infect 2009;58:259-265.

5. Colmenero JD, Jiménez-Mejías ME, Reguera JM, et al. Tuberculous vertebral osteomyelitis in the new millennium: still a diagnostic and therapeutic challenge. Eur J Clin Microbiol Infect Dis 2004;23:477-483.

6. Kamara E, Mehta S, Brust JCM, et al. Effect of delayed diagnosis on severity of Pott's disease. Int Orthop 2012;36(2):245-254.

7. Isik S, Johansen IS, Nielsen SL, et al. Characteristics and Clinical Outcome of Bone and Joint Tuberculosis From 1994 to 2011: A Retrospective Register-based Study in Denmark. CID 2015;61:554-62.

8. Jutte PC, van Loenhout-Rooyackers JH, Borgdorff MW, et al. Increase of bone and joint tuberculosis in The Netherlands. J Bone Joint Surg Br 2004;86:901-4.

9. Wibauxa C, Moafo-Tiatsopa M, Andreia I, et al. Changes in the incidence and management of spinal tuberculosis in a French University Hospital Rheumatology Department from 1966 to 2010. Joint Bone Spine 2013;80:516-519

10. Costello and Madewell. Clinical considerations and Imaging of bone tumors. In: Czerniak B, ed. Dorfman and Czerniak's Bone Tumors. Second ed. Philadelphia: Elsevier Inc (Saunders), 2015:57-96.

11. Burrill J, Williams CJ, Bain G, et al. A Radiologic Review. RadioGraphics 2007;27:1255-1273.

12. Russell DG, Cardona PJ, Kim MJ, et al. Foamy macrophages and the progression of the human tuberculosis granuloma. Nat Immuno 2009;10(9):943-8.

13. American Thoracic Society. Diagnostic Standards and Classification of Tuberculosis in Adults and Children. Am J Respir Crit Care 2000;161:1376-1395. 
14. Fukunaga $\mathbf{H}$, Murakami $\mathbf{T}$, Gondo $\mathbf{T}$, et al. Sensitivity of Acid-Fast Staining for Mycobacterium tuberculosis in Formalin-fixed Tissue. Am J Respir Crit Care Med 2002;166(7):994-997.

15. The Standards Unit, Microbiology Services, Public Health England. Investigation of specimens for Mycobacterium species. Bacteriology 2017;B40(7).

16. The Standards Unit, Microbiology Services, Public Health England. Investigation of bone and soft tissue associated with osteomyelitis. Bacteriology 2016;B42(2).

17. World Health Organisation. Global Tuberculosis Report 2016. Geneva, 2016.

18. National Institute for Clinical Excellence. Tuberculosis NICE guideline, 2016. http://www.nice.org.uk/guidance/ng33 (date last accessed 17 March 2017).

19. Boehme C, Nabeta P, Hillemann D, et al. Rapid Molecular Detection of Tuberculosis and Rifampin Resistance. N Engl J Med 2010;363:1005-1015.

20. World Health Organisation. Xpert MTB/RIF implementation manual. Technical and operational 'how-to' practical considerations. Geneva, 2014.

21. Maynard-Smith L, Larke N, Peters JA, et al. Diagnostic accuracy of the Xpert MTB/RIF assay for extrapulmonary and pulmonary tuberculosis when testing nonrespiratory samples: a systematic review. BMC Infectious Diseases 2014;14:709.

22. Tortoli E, Russo C, Piersimoni C, et al. Clinical validation of Xpert MTB/RIF for the diagnosis of extrapulmonary tuberculosis. Eur Respir J 2012;40:442-447.

23. Vadwai V, Boehme C, Nabeta P, et al. Xpert MTB/RIF: a New Pillar in Diagnosis of Extrapulmonary Tuberculosis? J Clin Microbiol 2011;49(7):2540-2545.

24. Held M, Laubscher M, Zar HJ, et al. GeneXpert polymerase chain reaction for spinal tuberculosis: an accurate and rapid diagnostic test. Bone and Joint J 2014;96(10):13661369.

25. Gu Y, Wanga G, Dong W, et al. Xpert MTB/RIF and GenoType MTBDRplus assays for the rapid diagnosis of bone and joint tuberculosis. IJID 2015;36: 27-30.

26. Ling DI, Zwerling AA, Pai M. GenoType MTBDR assays for the diagnosis of multidrugresistant tuberculosis: a meta-analysis. Eur Respir J 2008;32(5):1165-74.

27. Health Protection Agency, et al. National audit of TB diagnostic laboratories and description of molecular diagnostic provision. March 2012. 


\section{$\underline{\text { Tables }}$}

\section{Table 1}

Country-of-birth for patients diagnosed with TB, 2012-2014.

\begin{tabular}{|l|c|c|c|}
\hline \multirow{2}{*}{$\begin{array}{l}\text { Country of } \\
\text { birth }\end{array}$} & \multicolumn{3}{|c|}{$\begin{array}{c}\text { Time between symptom-onset and } \\
\text { referral to Specialist centre }\end{array}$} \\
\cline { 2 - 4 } & $\begin{array}{c}<6 \text { months } \\
(\mathrm{n})\end{array}$ & $\begin{array}{c}\geq 6 \text { months } \\
(\mathrm{n})\end{array}$ & $\begin{array}{c}\text { Unknown } \\
(\mathrm{n})\end{array}$ \\
\hline India & 5 & 3 & 3 \\
\hline Pakistan & 1 & 1 & 4 \\
\hline UK & 0 & 3 & 0 \\
\hline Afghanistan & 0 & 1 & 1 \\
\hline Bangladesh & 1 & 1 & 0 \\
\hline Fillipines & 0 & 1 & 0 \\
\hline Malaysia & 0 & 1 & 0 \\
\hline Nepal & 1 & 0 & 0 \\
\hline Somalia & 0 & 0 & 1 \\
\hline Sri Lanka & 0 & 0 & 1 \\
\hline $\begin{array}{l}\text { Not } \\
\text { documented }\end{array}$ & 1 & 0 & 1 \\
\hline
\end{tabular}


$\underline{\text { Table } 2}$

Anatomical site of TB infection for patients diagnosed with TB, 2012-2014.

\begin{tabular}{|c|c|c|c|c|}
\hline \multirow{2}{*}{ Site affected } & & \multicolumn{3}{|c|}{$\begin{array}{c}\text { Time between symptom-onset and referral } \\
\text { to Specialist centre }\end{array}$} \\
\hline & & $\begin{array}{l}<6 \text { months } \\
(\mathrm{n})\end{array}$ & $\begin{array}{l}\geq 6 \text { months } \\
(n)\end{array}$ & $\begin{array}{l}\text { Unknown } \\
\text { (n) }\end{array}$ \\
\hline \multicolumn{5}{|l|}{ JOINTS } \\
\hline & Hip & 0 & 1 & 1 \\
\hline & Knee & 2 & 1 & 0 \\
\hline & Ankle & 0 & 0 & 1 \\
\hline & Shoulder & 0 & 1 & 0 \\
\hline & Elbow & 1 & 1 & 1 \\
\hline & Wrist & 0 & 0 & 0 \\
\hline \multirow{2}{*}{\multicolumn{5}{|c|}{$\begin{array}{l}\text { LONG } \\
\text { BONES }\end{array}$}} \\
\hline & & & & \\
\hline & Foot/ toe & 0 & 2 & 1 \\
\hline & Hand/ finger & 0 & 2 & 0 \\
\hline & Arm & 0 & 0 & 0 \\
\hline & Leg & 0 & 0 & 1 \\
\hline \multirow{2}{*}{\multicolumn{5}{|c|}{$\begin{array}{l}\text { AXIAL } \\
\text { BONES }\end{array}$}} \\
\hline & & & & \\
\hline & Spine & 1 & 0 & 1 \\
\hline & Sacrum & 0 & 0 & 1 \\
\hline & Sternum & 1 & 0 & 0 \\
\hline \multicolumn{5}{|l|}{ MULTIFOCAL } \\
\hline & Including spine & 2 & 2 & 2 \\
\hline & $\begin{array}{l}\text { Not including } \\
\text { spine }\end{array}$ & 1 & 0 & 1 \\
\hline \multicolumn{5}{|l|}{ OTHER } \\
\hline & Soft tissue & 1 & 1 & 1 \\
\hline
\end{tabular}

